\title{
Aromatic Polyesters with Flexible Side Chains. 10. Studies on Biaxiality in Nematic Liquid Crystal of BC-n Polyester
}

\author{
Kai Fu, Masato Sone, Masatoshi Tokita, and Junji WAtanabe ${ }^{\dagger}$ \\ Department of Organic and Polymeric Materials, Tokyo Institute of Technology, \\ Ookayama, Meguro-ku 152-8552, Japan
}

(Received September 16, 2005; Accepted December 28, 2005; Published May 15, 2006)

\begin{abstract}
This paper reports a possibility of biaxiality in the nematic liquid crystal formed from aromatic polyesters, BC- $n$, which are composed of 1,4-dialkyl ester of pyromellitic acid and 4,4'-biphenol. In these BC- $n$ polyesters, as a general trend, the microsegregation of aromatic backbone and flexible side chains takes place and the nematic liquid crystal appears after these layered phases melt. X-Ray diffraction pattern from oriented nematic fiber shows unusual inner reflection with a large spacing around $20 \AA$ in addition to normal outer reflection with a spacing of $4.5 \AA$ on equatorial line, indicating that there are two types of packing correlation in a direction perpendicular to the director, $\mathbf{n}$, in other words, there is a biaxiality. Optical microscopic textures exhibit exclusively the two-brush disclinations with $|s|=1 / 2$, which also conforms to the biaxial nematic order. The spacing of inner equatorial reflection approximates to the layer spacing in the layered mesophase formed from the same homologues. This strongly suggests that the layered aggregation is still remained in the nematic liquid crystals, and its board-like shape is responsible for the biaxiality. [doi:10.1295/polymj.38.442]

KEY WORDS Aromatic Polyesters / Alkyl Side Chain / Liquid Crystal / Nematic / Biaxiality /
\end{abstract}

The rigid-rod aromatic polymers with long flexible alkyl side chains are one of the typical types of thermotropic polymers. ${ }^{1-5}$ The most interesting property of these materials is an ability to form layered structures in crystals and liquid crystals when the alkyl side chains reach a critical length. The layered mesophases are characterized by a packing of the rigid-rod main chains into layer with the molten side chains occupying a space between the layers. The driving force for an adoption of such a layered structure is a type of microphase separation of the aliphatic and aromatic domains and the liquid crystallinity is the result of a partial or total lack of positional order with respect to the main-chain packing within a layer and the conformational disordering of alkyl side chains between the layers. More recently, new type of mesophase, so called hexagonal columnar phase, has been found. ${ }^{6,7}$ This phase is especially interesting since a large number of molecules are included in a hexagonal lattice. The number of molecules increases from 7 to 12 with the increase of the carbon number of alkyl side chain from 8 to 14. Such a large number indicates a specific association of molecules. As the side chains are still conformationally disordered in this phase, a microsegregation structure has been proposed in which the aromatic main chains closely associate with each other to form a layer, but the layers are interconnected to form honeycombed network so as to surround the cylindrical side chain domains. ${ }^{7}$
In addition to these mesophases based on the microsegregation of two components, the polymers can also form nematic liquid crystal. Interesting is that there is a possibility of the biaxiality in its orientational field. Biaxial nematic phases are of great importance for extending the theory of liquid crystals, for understanding the molecular dynamic of mesogens, and for opening the possibility of fabricating new types of liquid crystal materials. In this study, we treat the nematic phase in BC- $n$ polyesters, which are prepared by the esterification of 1,4-ditetraalkyl ester of pyromellitic acid and 4,4'-biphenol,

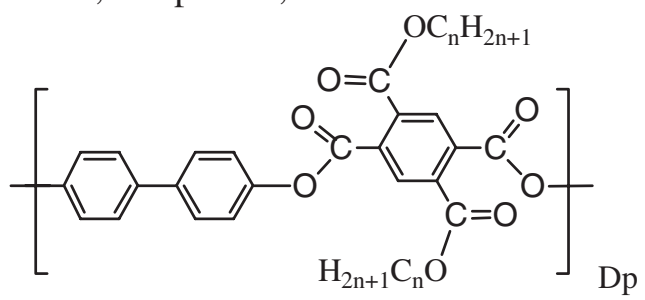

BC-n

$$
n=6,8,10,12,14,16,18,20
$$

and discuss the possibility of its biaxiality. This study will stimulate future recognition of biaxial nematic phases in the polymer system.

\section{EXPERIMENTAL}

Synthesis of BC- $n$ polymers and their characterization have been described in a previous paper. ${ }^{3}$

†To whom correspondence should be addressed (Tel: +81-3-5734-2633, Fax: +81-3-5734-2888, E-mail: jwatanab@ polymer.titech.ac.jp). 
Optical microscopic observations of the liquid crystalline textures were made with an Olympus BX50 polarizing microscope equipped with a Mettler FP-82 hot stage. Differential scanning calorimetric (DSC) measurements were carried out with a Perkin Elmer Pyris I at a scanning rate of $10^{\circ} \mathrm{C} / \mathrm{min}$ under a flow of dry nitrogen. Wide angle X-ray diffraction (WAXD) measurement was performed using a Rigaku-Denki RU-200 BH with Ni-filtered $\mathrm{CuK}_{\alpha}$ radiation. Temperature of the sample was controlled by a Mettler FP- 82 hot stage mounted in the beam path. The film to specimen distance was determined by calibration with silicon powder.

\section{RESULTS}

We first describe the phase behavior and phase structure in the typical polymer, BC-14. In order to avoid any thermal prehistory, the sample was first heated to the isotropic melt before use. DSC scan of melt-pressed film showed two small transitions on heating and cooling, respectively. ${ }^{3}$ On heating, the transition temperatures are 123 and $179^{\circ} \mathrm{C}$, which are assigned to $T_{1}$ and $T_{\mathrm{i}}$, respectively. On cooling, the corresponding transitions were observed at 110 and $164^{\circ} \mathrm{C}$. Here, $T_{\mathrm{i}}$ is the isotropization temperature of the nematic phase, and $T_{1}$ the transition temperature from the nematic phase to the layered mesophase. Transition enthalpies are small for these and are around $0.1 \mathrm{kcal} \mathrm{mol}^{-1}$.

Figure 1 shows the optical microscopic texture of the nematic liquid crystal. The typical Schlieren texture can be seen, confirming the nematic order. Interesting is that the Schlieren texture includes exclusively the two-brush disclinations with $|s|=1 / 2$, which is certainly an indication of biaxiality ${ }^{8}$ as supported by the topological considerations of Mermin ${ }^{9}$ and the

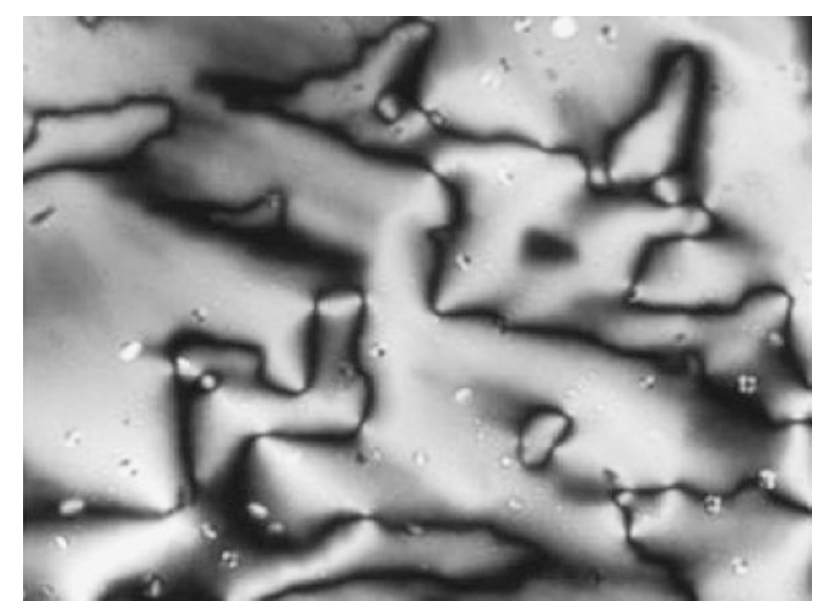

Figure 1. Schlieren texture exhibited by the nematic phase of BC-14 polyester. Only two-brush disclinations, $|s|=1 / 2$, are observed.

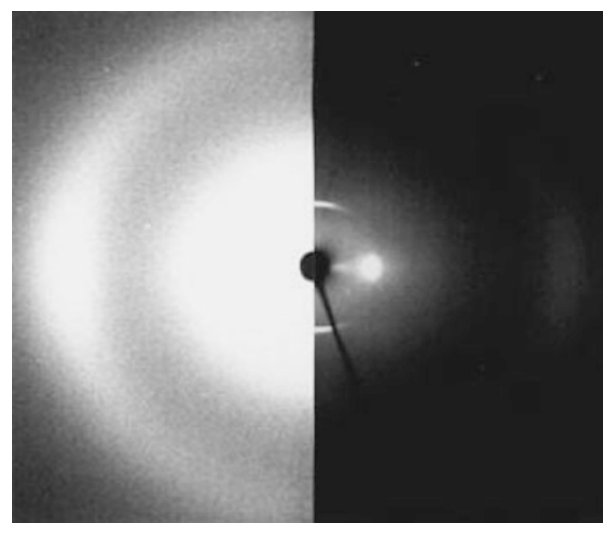

Figure 2. X-Ray photograph of the nematic fiber of BC-14 recorded at $140{ }^{\circ} \mathrm{C}$. Fiber axis is in the vertical direction.

computer simulations by Zannoni, et al. ${ }^{10}$ The freestanding film also shows only $|s|=1 / 2$ defects, which eliminates possibility of biaxiality produced by a glass surface.

X-Ray diffraction pattern was carefully observed to obtain confirmatory evidence of the biaxial nature. Figure 2 shows the X-ray pattern taken for the oriented nematic fiber of BC-14 which was spun in the nematic field. It is well known that a uniaxial nematic gives rise to two liquid-like $\mathrm{X}$-ray diffraction maxima, one corresponding to mean repeat distance along the director axis and the other to the mean lateral intermolecular spacing. In a case of the biaxial nematic phase, one expects three maxima, ${ }^{11}$ which are just observed in X-ray pattern of Figure 2. Among three distinct reflections, the two appear along an equatorial line and the one along a meridional line. These can be definitely recognized from intensity profiles observed along the equatorial line (Figure $3 \mathrm{a}$ ) and along the meridional line (Figure $3 b$ ). The meridional reflection appears as arc with a spacing of $15.3 \AA$, which corresponds to the average repeat distance of polymer backbone. ${ }^{3}$ The value is somewhat smaller than $16.5 \AA$, the length of the repeat unit in fully extended conformation. The two broad equatorial reflections resulting from the lateral packing structure, appear in the small-angle (inner) and wide-angle (outer) regions. The inner reflection has a spacing of $23.4 \AA$, while the outer reflection has a spacing of $4.5 \AA$. The outer reflection is usual for the nematic liquid crystal since its spacing of $4.5 \AA$ corresponds to the lateral packing distance of aromatic LC molecules. The additional inner equatorial reflection, hence, shows the possibility of another order in lateral packing, i.e. biaxial orientation of molecules.

Similar optical texture and X-ray pattern are observed for the nematic phases of all other BC- $n$ polyesters with $n$ of 6 to 20. In Figure 4, the spacings of the equatorial and meridional reflections are plotted against the carbon number of alkyl side chain, $n$, as 
(a)

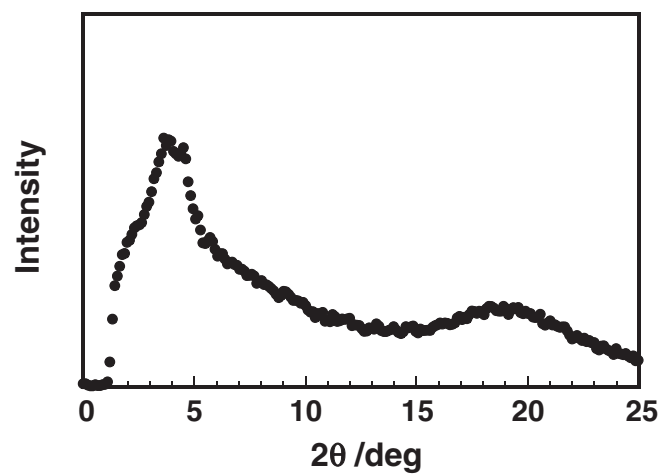

(b)

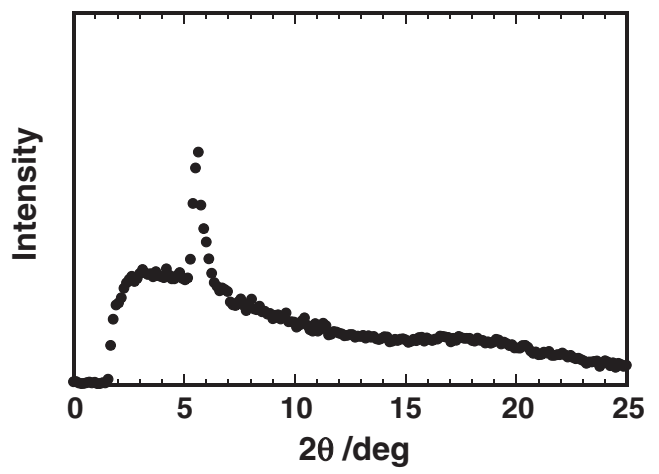

Figure 3. Intensity profiles of the oriented X-ray pattern of

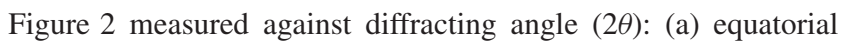
scan and (b) meridional scan.

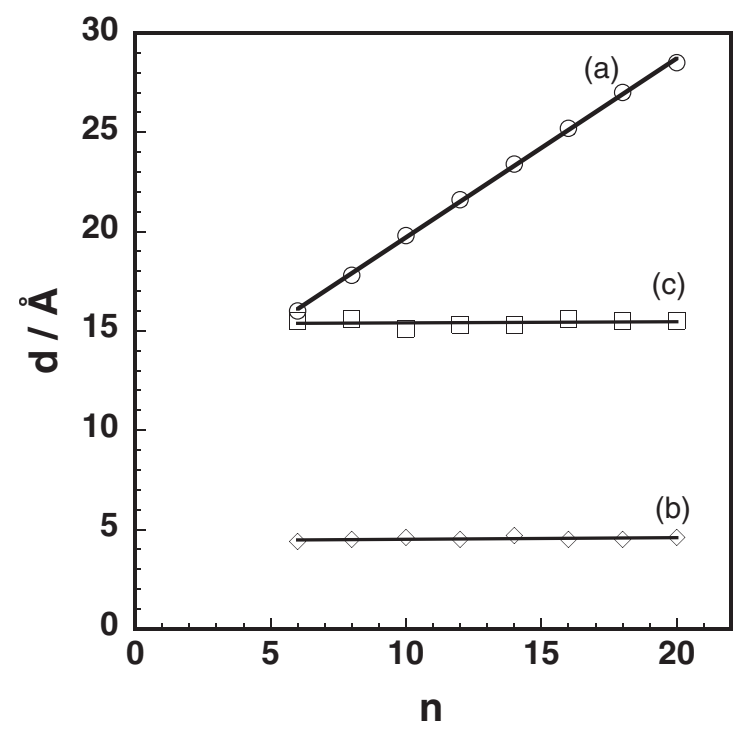

Figure 4. Variation of the spacings of (a) the small-angle equatorial reflection (circle), (b) the wide-angle equatorial reflection (lozenge) and (c) the (001) meridional reflection (square) with the alkyl side chain length, $n$.

collected from the nematic phase at $120-140{ }^{\circ} \mathrm{C}$. The spacing of the inner equatorial reflection increases monotonically from 16.0 to $28.5 \AA$ when the value of $n$ increases from 6 to 20, whereas the spacings of

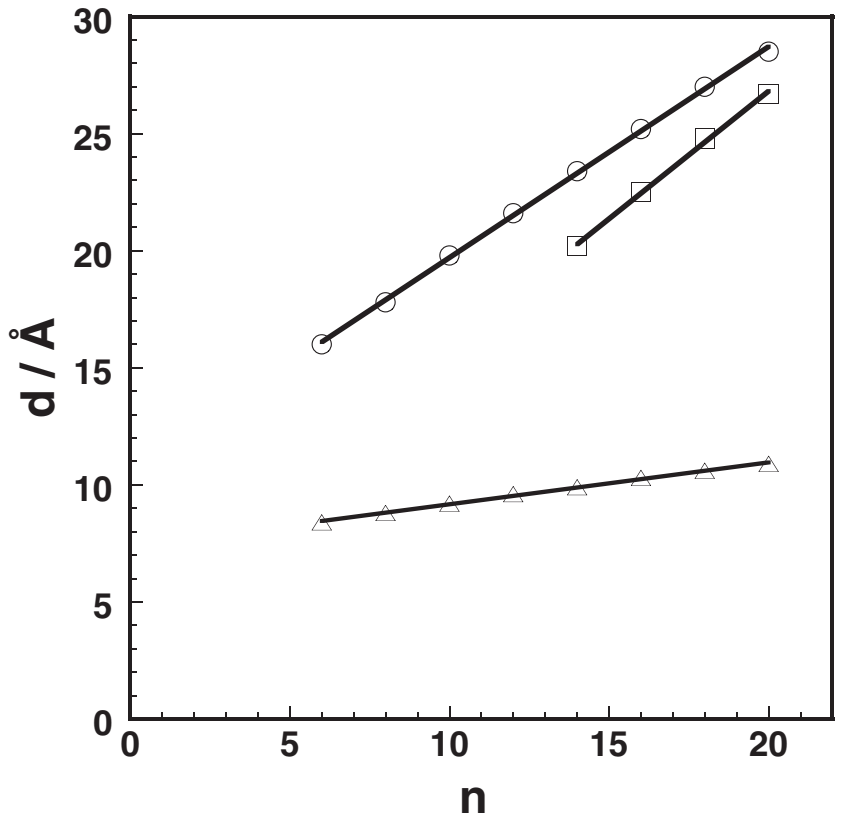

Figure 5. Comparison of the spacing of (a) the small-angle equatorial reflection (circle) with (b) the averaged molecular diameter (triangle) and (c) the layer spacing of the lower temperature layered mesophase (square) (refer to the text).

the meridional reflection and outer equatorial reflection are almost constant at around 15.5 and $4.5 \AA$, respectively.

\section{DISCUSSION}

Let's first imagine an ordinary nematic phase, in which each molecule behaves as a kinetic unit and therefore rotates freely around its long axis. If such an ordinal phase is to occur in this system, lateral spacing should correspond to the average diameter of molecule. The spacings can be calculated under the assumption of the density of $1 \mathrm{~g} / \mathrm{mL}$, and in Figure 5 , are plotted against $\mathrm{n}$ to compare with the spacings of the inner equatorial reflection. These spacings monotonically increase with the increase of $n$ as is observed in the spacings of the inner equatorial reflections, but their absolute values are remarkably smaller. Thus, we shortly conclude that no ordinal nematic liquid crystal is formed here.

It is interesting to say that the spacing of inner equatorial reflection approximates to the layer spacing of layered mesophase which is formed in the lower temperature region than the nematic phase. ${ }^{3}$ The layer spacings collected from $\mathrm{BC}-14, \mathrm{BC}-16, \mathrm{BC}-18$ and BC-20, are plotted against $n$ in Figure 5. Both roughly correspond to each other although the spacings in the nematic phase are somewhat larger than the layer spacings. A similar result has been obtained for the nematic phase of polyamides with alkyl side chains as reported by Ebert, et al. ${ }^{12}$ 


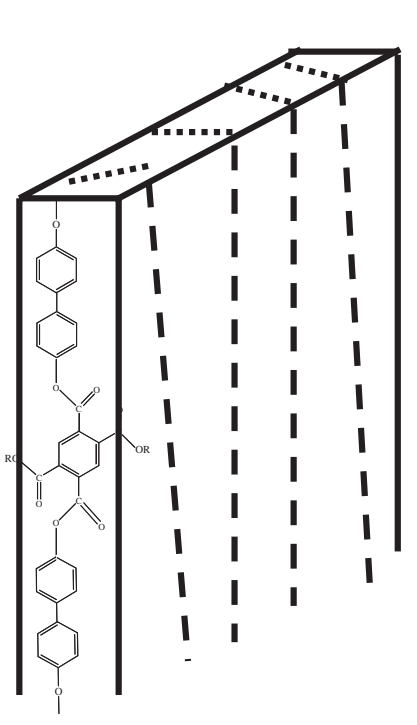

(a)

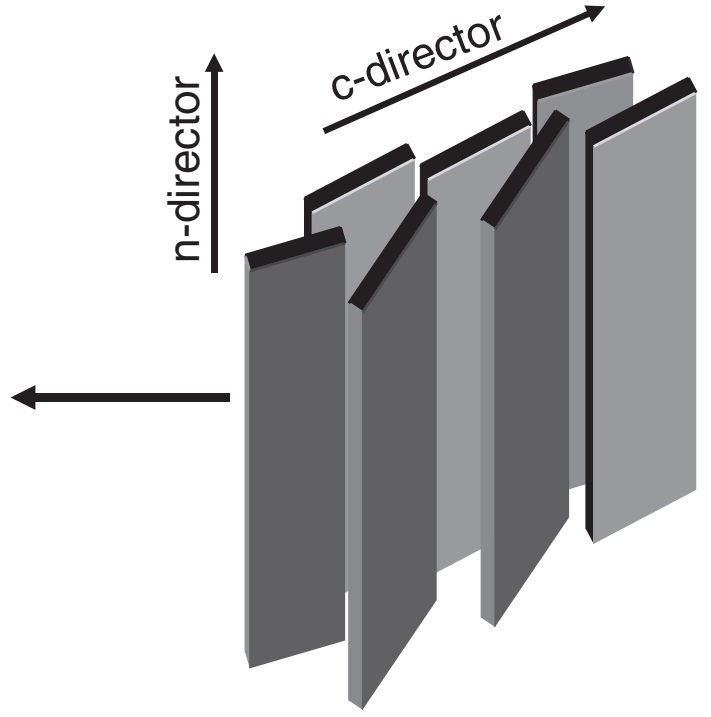

(b)

Figure 6. Illustration of the biaxial nematic liquid crystal. Here, the main chains are associated with each other to form the layer as in the lower temperature layered mesophase or crystal (refer to (a)), but the layers are packed with a greater degree of disorder as in (b). To produce such a disorder, the layer may be constructed with only short-range correlation length. Then, the additional director, c, exists along the plane of layer.

In these characteristic polymers, the aromatic main chain and the aliphatic side chain micro-segregate from each domain. The basic segregation takes place in the layered form in which main chain backbones are packed into a layer and such a main chain layer is sandwiched by the aliphatic side chains. This characteristic aggregation leads to the layered and hexagonal columnar mesophases as stated in Introduction. The present nematic liquid crystals can be understood on the same basis of this type of segregation, such that the main chains are still associated with each other to form a layer, but the layers are packed with a greater degree of disorder. To produce such a disorder, the layer may be constructed with only short-range correlation length although at present we have no information on its length. Then, the additional director lies in the plane of the layer as illustrated in Figure 6. Thus, the larger spacing comes from the layer-like packing while the smaller one corresponds to the lateral packing distance of main chain within a layer. The density of nematic phase can be roughly calculated based on the rectangular lattice. They are ranged from 0.90 to $0.95 \mathrm{~g} / \mathrm{mL}$, which are reasonable for the mesophase.

The orientational order parameters, $S$

$$
S=\frac{\left(3\left\langle\cos ^{2} \beta\right\rangle-1\right)}{2}
$$

can be determined from an azimuthal scan for the two equatorial reflections of Figure 2 according to the following equation, ${ }^{13}$

$$
\left\langle\cos ^{2} \beta\right\rangle=\frac{\int_{0}^{\pi / 2} I(\beta) \cos ^{2} \beta|\sin \beta| d \beta}{\int_{0}^{\pi / 2} I(\beta)|\sin \beta| d \beta}
$$

Here, $\beta$ is the azimuthal angle taken from the equatorial line. The order parameter is 0.46 from the outer reflection, while it is 0.56 from the inner one. The difference is not negligible, confirming that there are two types of the orientational order, i.e., those with respect to polymer chain and layer. The higher order parameter of the layer orientation may be reasonable if the molecules are packed within a layer with some orientational disordering.

It is reliable that the biaxiality is produced by a hindered rotation of constituent molecules. ${ }^{8,11,14-16} \mathrm{~A}$ convenient way to obtain the biaxial nematic phase is to bridge the gap between rod-like and disk-like molecules, i.e., to prepare a mesogen that combines the features of the rod and the disk. These are called shape-biaxiality. The banana shaped molecules which have been studied extensively, also belong to this type of molecules. ${ }^{17,18}$ In the present system, the induction of biaxiality may be due to the aggregation of rod molecules into a layer. This is completely different from the systems so far reported, suggesting another method to attach the biaxiality to nematic liquid crystals. Finally, it should be noted that simple aromatic polyesters without any side chains may form the biaxial nematic LC as well. ${ }^{13,19,20}$ 


\section{CONCLUSIONS}

The nematic liquid crystal formed from the BC- $n$ polyesters showed the characteristic X-ray pattern including two equatorial reflections. One is the normally observed reflection with a spacing of $4.5 \AA$ and another is the unusual reflection with large spacing ranging from 20 to $30 \AA$. These two reflections show the biaxiality of the nematic liquid crystal in which there are two types of packing correlation in a direction perpendicular to the director, n. Optical microscopic textures exhibited exclusively the twobrush disclinations with $|s|=1 / 2$, also supporting the biaxial nematic order. The spacing of inner equatorial reflection approximates to the layer spacing in the layered mesophase formed from the same homologues. The board-like shape due to the layered aggregation of polymer molecules was considered to be responsible for the biaxility.

\section{REFERENCES}

1. J. Watanabe, H. Ono, I. Uematsu, and A. Abe, Macromolecules, 18, 2141 (1985).

2. M. Ballauff, Angew. Chem., Int. Ed., 28, 253 (1989).

3. J. M. Rodrigues-Parada, R. Duran, and G. Wegner, Macromolecules, 22, 2507 (1989).

4. M. Ebert, O. Herrmann-Schenherr, J. Wendorf, H. Ringsdorf, and P. Tschirner, Liq. Cryst., 7, 63 (1990).

5. J. Watanabe, B. R. Harkness, H. Ichimura, and M. Sone, Macromolecules, 27, 507 (1994).

6. J. Watanabe, N. Sekine, T. Nematsu, M. Sone, and H. R. Kricheldorf, Macromolecules, 29, 4816 (1996).
7. K. Fu, M. Sone, M. Tokita, and J. Watanabe, Polym. J., 34, 291 (2002).

8. S. Chandrasekhar, S., G. G. Nair, D. S. Shankar Rao, and S. Krishna Prasad, Liq. Cryst., 24, 67 (1998).

9. N. D. Mermin, Rev. Mod. Phys., 51, 591 (1977).

10. C. Chiccoli, I. Feruli, O. D. Lavrentovich, P. Pasini, S. V. Shiyanovskii, and C. Zannoni, Phys. Rev. E, 66, 030701 (2002).

11. a) K. Praefcke, B. Kohne, B. Gundogan, D. Demus, S. Diele, and G. Pelzl, Mol. Cryst. Liq. Cryst. Lett., 7, 27 (1990).

b) K. Praefcke, B. Kohne, B. Gundogan, D. Singer, D. Demus, S. Diele, G. Pelzl, and U. Bakowsky, Mol. Cryst. Liq. Cryst., 198, 393 (1991).

12. M. Ebert, O. Hermann-Schonherr, J. H. Wendorff, H. Ringsdorf, and P. Tschirner, Makromol. Chem. Rapid Commun., 9, 445 (1988).

13. G. R. Mitchell and A. H. Windle, in "Developments in Crystalline Polymers,” D. C. Bassett, Ed., Elsevier, London, 1988, Vol. 2.

14. G. R. Luckhurst, Thin Solid Films, 393, 40 (2001).

15. a) L. Omens, B. A. Timini, T. Gelbrich, M. B. Hursthouse, G. R. Luckhurst, and D. W. Bruce, Chem. Commun., 2248 (2001).

b) R. W. Date and D. W. Bruce, J. Am. Chem. Soc., 125, 9012 (2003).

16. B. R. Acharya, A. Primak, T. J. Dingemans, E. T. Samulski, and S. Kumar, Pramana, 61, 231 (2003).

17. T. Niori, T. Sekine, J. Watanabe, T. Furukawa, and H. Takezoe, J. Mater. Chem., 6, 1231 (1996).

18. R. Pratibha, N. V. Madhusudana, and B. K. Sadashiva, Science, 288, 2184 (2002).

19. J. Watanabe, L. YuQing, H. Tsuchiya, and H. Takezoe, Mol. Cryst. Liq. Cryst., 346, 9 (2002).

20. B. Mettout, P. Toledano, H. Takezoe, and J. Watanabe, Phys. Rev. E, 66, 031701-1 (2002). 